\title{
Exploring the Solution of Bionic Prosthesis Lacking Perceptual Feedback System: Using Invasive and Non-invasive Techniques
}

\author{
Xiaohan Hong ${ }^{1}$ \\ ${ }^{1}$ Kaifeng High School, Henan Province,475000, China
}

\begin{abstract}
The bionic prosthesis is a more advanced research direction in the prosthesis, but there are still many shortcomings. One of them is the lack of an effective perceptual feedback system, which included the feedback of position, force, texture, roughness, temperature, and other environmental information, as well as the information perception ability of the prosthesis itself[1]. This defect limits the normal operation of the prosthesis and endanger the safety of users. By analyzing the sensory feedback mechanism of the human body, it is found in this paper, the main reason for the lack of an effective perceptual feedback system of the bionic prosthesis is that there is no structure connected with the internal nerves of the human body in the mechanical prosthesis. Therefore, the perceptual signals collected by artificial sensors cannot be transmitted to the cerebral cortex by invasive method, or lack specific in vitro devices to stimulate patients to produce specific perceptual feedback by non-invasive methods. At present, through the use of invasive and non-invasive technology, we have mastered the form of electrical stimulation, vibration stimulation, and other forms of perceptual feedback. Due to the larger amount of feedback information, shorter training time than other methods, non-invasive electrical stimulation feedback has become a promising research area in this field.
\end{abstract}

\section{Introduction}

The bionic prosthesis is a prosthesis that combines bionics and electronic function, that is, it uses an electronic function to strengthen the biological function of the prosthesis. Amputees can control the movement of the bionic prostheses through their own will and muscles, which is a more advanced prosthetic type in the field of prosthetics. The existing bionic prosthesis feedback form is single. The patient can only control the prosthesis through their own visual perception to interact with the external environment, because of the lack of effective perceptual feedback, the operation experience is poor, especially in the environment with poor illumination conditions. Adding sensory function to the prosthesis, imitating human's original sense of touch, temperature, pain, and so on, is a way to solve the above problems. Also, it can help patients reduce limb pain to a large extent.

The author chooses the problem, the lack of an effective perception feedback system for bionic prosthesis, to explore. The study aims to explore how to solve the problem of the lack of an effective perceptual feedback system of the bionic prosthesis by non-invasive and intrusive methods. This paper will be carried out by exploring the following questions: Firstly, by comparing the human sensory feedback mechanism, exploring the reasons for the lack of an effective perceptual feedback system for the bionic prosthesis. Secondly, How to solve this problem by intrusive and non-intrusive methods.
Thirdly, current possible solutions and still existing problems among them. Fourthly, the main research directions in this field.

\section{Signal feedback mechanism}

\subsection{Human Sensory Feedback Mechanism}

In medicine, sensory production is usually described as the result of the interaction of three parts: receptor or sensory organ, nerve conduction pathway, and sensory center. The general process: various stimuli in the internal and external environment of the body first act on different receptors or sensory organs, and they are converted into nerve impulses, which are integrated or analyzed through special neural pathways to specific areas of the cerebral cortex to produce corresponding feelings. Among them, the human body's sensations can be roughly divided into body and visceral sensations, vision, hearing, balanced sensation, smell, and taste[6]. The feeling that needs to be feedback in bionic limbs is mainly somatic sensation.

\subsection{Specific Feedback Mechanism of Somatic Sensory}

The somatic sensation is derived from the information provided by various receptors throughout the body, mainly sensing touch-pressure (recognizing the texture, shape, texture, etc.), position and motion (proprioceptive),

\footnotetext{
*Corresponding author: ShiLiShuang@cas-harbour.org
} 
and temperature (cold, heat) and nociceptive (pain and itching). The body receives different stimuli through the skin and its accessory receptors to produce various types of sensation. It is generally believed that somatosensory includes two categories: shallow sensation and deep sensation. Shallow sensation has touch-pressure sensation, temperature sensation, and pain sensation; the deep sensation is proprioceptive sensation, which mainly includes position sensation and motor sensation. As shown in Figure 1, the primary afferent neurons of somatosensory are located in the dorsal root ganglion or the brain ganglion, and their peripheral processes are connected to the receptors; The central process enters the spinal cord and the brain stem, and issues two types of branches. One kind is that forming reflex arc by connecting interneurons with motor neurons directly or indirectly at different levels (see figure 2), to complete all kinds of reflections; the other one is that being relayed by multistage neurons (see figure 3 ) to project to the cerebral cortex (see figure 4) and produce different sensations[6].

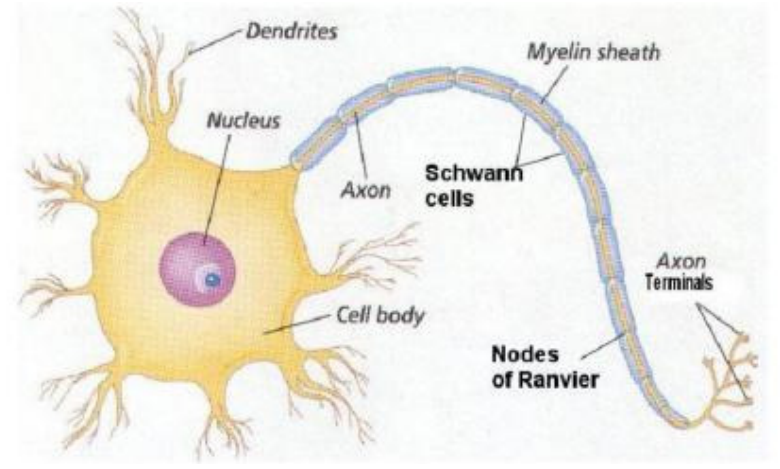

Fig. 1. Schematic diagram of the structure of biological neurons.

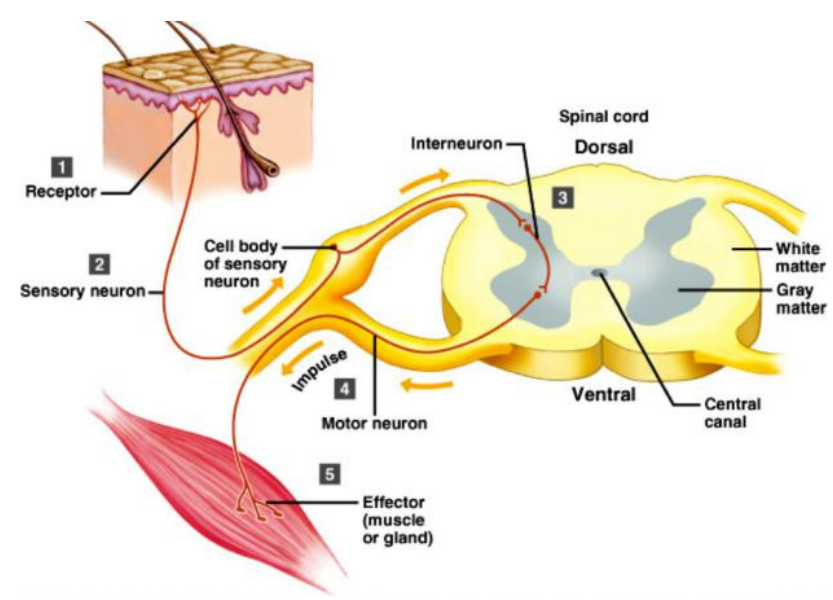

Fig. 2. Reflector Arc diagram.

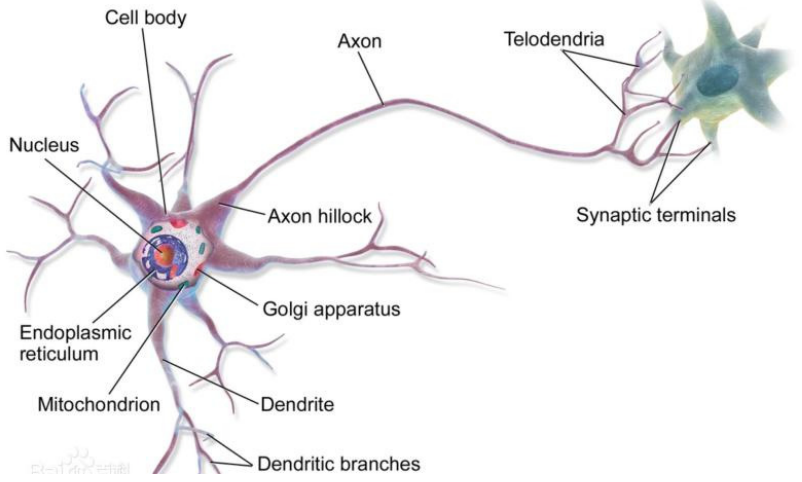

Fig. 3. Multistage neuron diagram.

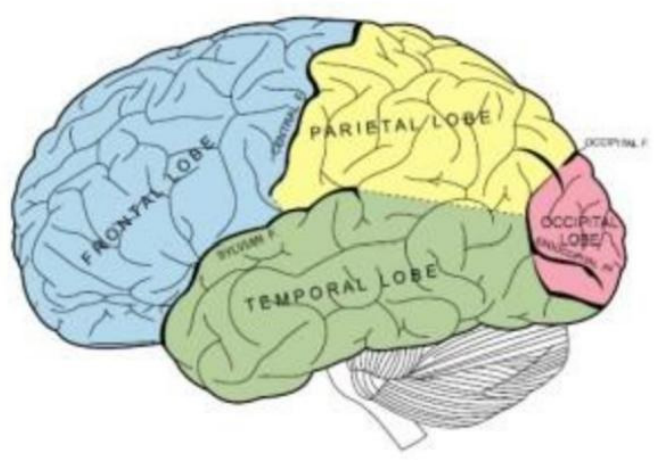

Fig. 4. Schematic diagram of lobulation in the cortex of the large brain.

Therefore, due to the damage of the nerve conduction pathway in the stump, the amputation patients cannot form a complete reflex, so it is impossible to form a corresponding feeling through external stimulation. However, because the knowledge of neural decoding is still not mastered, there are great problems in reconstructing or reducing the nerve pathway of the stump of amputation patients. Besides, the specific reason for limited neural decoding knowledge. During the conversion of external stimuli to nerve action potentials, the receptor not only converts energy, but it also transmits information about environmental changes contained in stimuli to new electrical signal systems, which is the sequence of action potentials. In other words, encoding (decoding) operations and the brain are based on these electrical signal sequences to access external understanding in the world, while people have not understood the principle of coding.

\section{Existing Solutions}

In contrast to the human sensory feedback mechanism, the main reason for the lack of an effective sensing feedback system is that there is no structure connected with the internal nerves in the mechanical prosthesis so that it is impossible to transmit sensory signals collected by artificial sensors to the cortex; or lacking specific in vitro devices which stimulate patients by non-invasive ways to generate specific perceptual feedback. The lack of effective perceptual feedback will cause great inconvenience to the life of amputees. For example, at 
night or in a darker environment, amputation patients cannot gain surrounding environment information through prostheses, thus greatly increasing the burden of the eyes. Information cannot be obtained only by touch or other ways.

At present, many experts and scholars have carried out in-depth research in this field, providing many possible solutions, which are summarized below:

\subsection{Perceived signals}

Perceived signals are the information of the external environment collected and processed by the artificial sensor. The principle of the sensor is mainly divided into resistance, capacitance, photoelectric, piezoelectric, and so on [1-3]. Resistive tactile receptors: the basic principle of the resistive tactile sensor is that when strain or pressure is applied to the sensor, the deformation inside the sensor will cause the change of resistance in the sensor, and the information of external strain and pressure can be obtained by measuring the change of resistance, for example, Yin and other developed resistance microfluidic shear force sensor (see figure 5) [4]. However, its disadvantages are high cost and low precision. Capacitive tactile receptors: the basic principle of capacitive tactile receptors is that when the pressure is received, the distance between the two parallel conductive layers that make up the sensor will be reduced to deform the dielectric material, changing sensor capacitance. The basic principle of the photoelectric tactile sensor is that the light emitted from the sensor is reflected by the surface of the external object and collected and processed by the optical detector inside the sensor. For example, Totaro and others develop the stretchable capacitive sensor composed of conductive fabric and elastic layer (see figure 6)[5], while the disadvantage is that the size is large and the detection range is small. The basic principle of the piezoelectric tactile sensor is that after the sensor is under pressure, the internal charge distribution will also change, and the pressure can be calculated by measuring the change of voltage caused by the change of charge distribution.

These sensors can provide accurate sensing signals for amputees as much as possible so that amputees can receive feelings closer to the natural limbs and as much external information as possible. At present, the main problems faced by sensors are high cost; poor material flexibility, making patients wear discomfort; low accuracy; less detectable signals, etc.

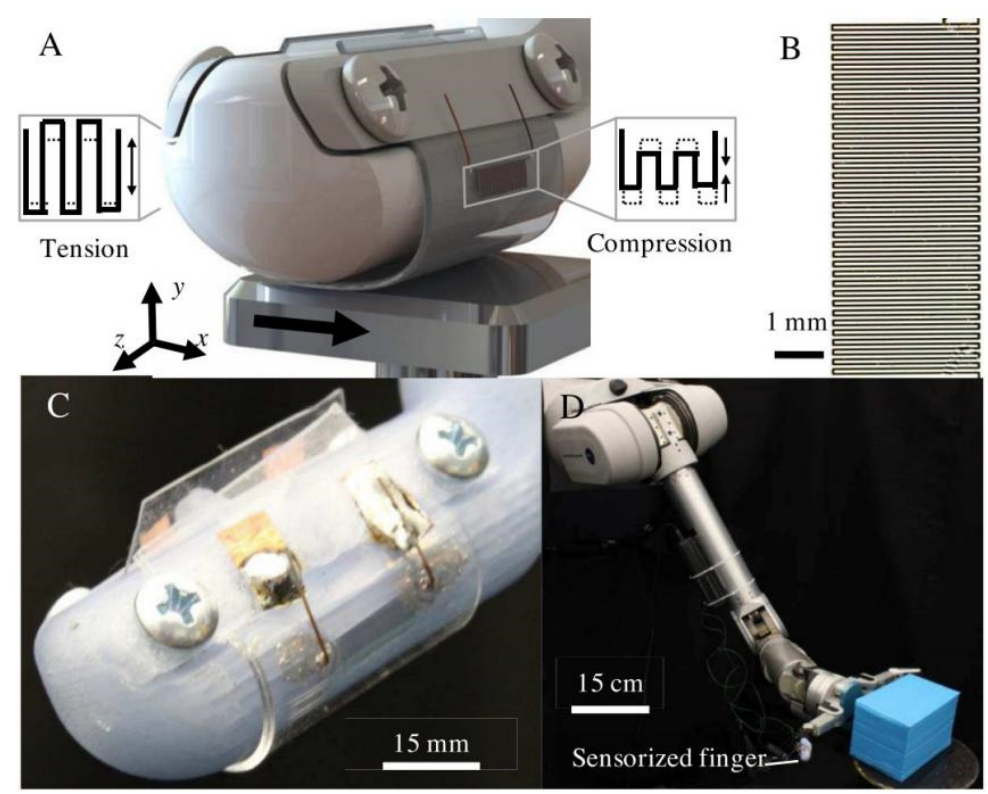

Fig. 5. Yin schematic diagram of resistance microfluidic shear force sensor[4].

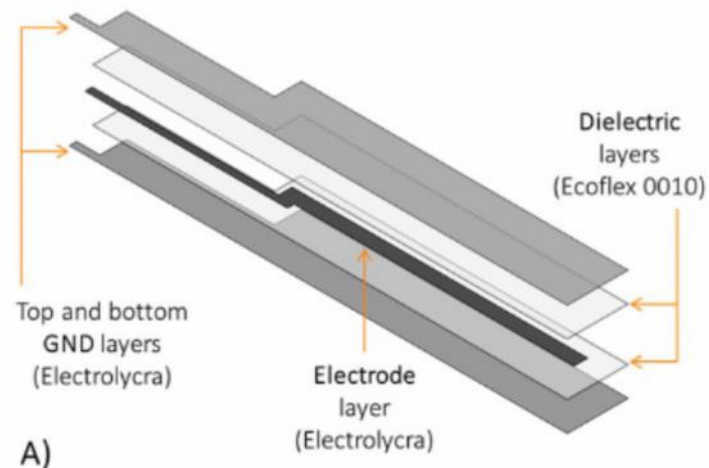

Fig. 6. Totaro schematic diagram of capacitive sensor[5]. 


\subsection{Perceptual feedback patterns}

The sensing signals collected and processed by the above sensors are transmitted to amputees by certain perceptual feedback patterns, which can help amputees get sensory feedback similar to the natural limb. Perceptual feedback can be divided into intrusive and non-intrusive two patterns, the following is a brief introduction.

\subsubsection{Non-invasive}

The most commonly used non-invasive methods include Mechanotactile feedback, Vibrotactile feedback, Electrotactile feedback, Hybrid stimulation, and Auditory stimulation[2]. Non-invasive electrical stimulation is to adjust the waveform, amplitude, frequency, and duty cycle of the feedback current to make the wearer feel different. Non-invasive vibration stimuli give the wearer different feelings through different vibration frequencies or intensities.

Current issues for non-intrusive perceived feedback: Firstly, how to make the non-invasive stimulation, on the skin of the residual limb or elsewhere on the body, somatotopically matched and perceived as touching of the lost fingers or wherever placement of the sensors[2]. Secondly, making non-intrusive feedback devices more comfortable to wear. Thirdly, the perceived feedback system generally has large power consumption and long response time.

\subsubsection{Invasion}

The invasive electrical stimulation feedback system implants the stimulation electrode at a certain part of the patient's body through surgery, so that the implanted electrode is directly or indirectly connected with the internal nerve, and the perceptual information is transmitted to the internal nerve through the implanted electrode in a specific mode of current [1]. In theory, patients can get the same perceptual feedback as normal people, but due to the risk of surgery, long postoperative recovery time, and the limitation of neural decoding knowledge, only a few researchers are studying. It is not a trend of research.

\section{Conclusion}

The purpose of making bionic prostheses is to replace natural limbs and minimize the inconvenience caused by amputation. Therefore, the goal of the bionic prosthesis is to be as close as possible to the natural limb, whether in function or shape. Therefore, the general development direction of the bionic prosthesis is low cost and more functions of natural limbs. In addition, the material can be closer to natural arms and lighter. At present, due to the outstanding contribution of researchers, the research results in this field are very rich, including the Michelangelo ${ }^{\circledR}$-Hand Advanced Prosthesis System (Otto Bock Healthcare Products GmbH, D), the iLimb
Hand (Touch Bionics, UK), the Vincent Hand (Vincent Systems GmbH, D), the be-bionics-Hand (RSLSteeper, UK), etc. The perceptual feedback technology of bionic prosthesis has been developed for many years, but there are still many problems to be solved: How to make the feedback device more miniaturized, universal, and comfortable; how to reduce the training time of the wearer; how to improve the resolution of the feedback information, etc. Also, because of the large amount of feedback information, the short training time, and the small injury to the patient, the non-invasive electrical stimulation feedback has become the research hotspot of bionic prosthesis perception feedback technology.

\section{References}

1. B. Zhu, Y. Chu, X. Zhao, etc. Research progress of perception and feedback technology in prostheses.Journal of Biomedical Engineering, 36(6) (Dec. 2019)

2. 2P. Svensson, U. Wijk, A. Bjorkman, et al. A review of invasive and non-invasive sensory feedback in upper limb prostheses. Expert Rev Med Devices, 14(6): 439-447 (2017).

3. B. Stephens-Fripp, G. Alici, R. Mutlu. A review of non-invasive sensory feedback methods for transradial prosthetic hands. IEEE Access, 6: 6878-6899 (2018).

4. J. Yin, P. Aspinall, V.J. Santos, et al. Measuring dynamic shear force and vibration with a bioinspired tactile sensor skin. IEEE Sens J, 18(9):3544-3553 (2018).

5. M. Totaro, T. Poliero, A. Mondini, et al. Soft smart garments for lower limb joint position analysis. Sensors, 17(10): 2314- 2336 (2017).

6. D. Zhu, T. Wang. Physiology. Ninth edition. Beijing: People's Medical Publishing House (2019). 